\title{
VIDEO TEST TO EVALUATE DETECTION PERFORMANCE OF DRIVERS WITH HEMIANOPIA AND QUADRANOPIA: PRELIMINARY RESULTS
}

\author{
Alex R. Bowers ${ }^{1,2}$, Karen Jeng ${ }^{3}$, Eli Peli ${ }^{1,2}$, Laura Werner $^{2}$ \& Amy Doherty ${ }^{1}$ \\ ${ }^{1}$ Schepens Eye Research Institute, Mass Eye and Ear, Harvard Med School, Boston, \\ Massachusetts, USA \\ ${ }^{2}$ New England College of Optometry, Boston, Massachusetts, USA \\ ${ }^{3}$ UMDNJ-Robert Wood Johnson Medical School, Piscataway, New Jersey, USA \\ Email: alex_bowers@meei.harvard.edu
}

\begin{abstract}
Summary: The ability of individuals with hemianopia to compensate for their vision impairment by eye/head scanning to detect hazards in their non-seeing (blind) hemifield varies widely in both simulator and on-road tests. Conventional visual fields tests do not reflect this variability, while simulator and on-road tests are time-consuming and expensive. We therefore developed a simple, 15-minute video-based pedestrian detection test suitable for implementation on a desktop computer and monitor. The test was found to be sensitive to detection deficits in both hemianopia and quadranopia, and predictive of detection performance in a driving simulator. Our preliminary findings suggest that the test provides a simple method of measuring detection ability relevant to driving which may be useful both as a screening test and as an evaluation tool for rehabilitation devices and training.
\end{abstract}

\section{BACKGROUND AND OBJECTIVES}

Hemianopia is the loss of half of the visual field on the same side in both eyes that commonly occurs due to stroke and traumatic brain injury. Twenty-two states prohibit people with hemianopia from driving as they do not meet the minimum field extent requirement (e.g. $120^{\circ}$ in Massachusetts) (Peli, 2002). However, in other states they may be permitted to drive, and in some countries (e.g., Belgium, Netherlands, Switzerland, UK, Canada) persons with hemianopia may be licensed after taking a specialized road test (Dow, 2011; DVLA Drivers Medical Group, 2011; Yazdan-Ashoori \& ten Hove, 2010).

Visual field extent is usually measured under simple visual conditions: detection of a white light on a plain uniform background while looking at a central point. However, in driving, detection of objects occurs against complex backgrounds and the driver is free to make eye and head movements. People with hemianopia may be able to compensate for the field loss by eye and head scanning; however, conventional visual field tests can not reflect such ability. Although individuals with complete hemianopia may appear to have identical amounts of vision loss on a visual fields test, their ability to use their remaining vision in more complex tasks, such as detection of objects when walking and driving, varies widely (Bowers et al., 2009; Iorizzo et al., 2011; Papageorgiou et al., 2012). For example, in a driving simulator study, detection rates for pedestrians that appeared on the side of the blind hemifield varied from as little as $6 \%$ to as high as $100 \%$, yet all the participants had complete hemianopia, no significant cognitive impairment and no spatial neglect (Bowers et al., 2009). 
Thus there is a need for a test that measures an individual's ability to use remaining vision for a task that is relevant to driving and uses more real-world conditions than conventional visual fields tests, but is simple enough to be used during routine screening or as an outcome measure in multicenter clinic-based studies. One possibility would be to use a driving simulator (Bowers et al., 2009; Bronstad et al., 2011; Papageorgiou et al., 2012); however, use of a simulator is expensive and time consuming, and requires specialized equipment and expertise. We developed a flexible, video-based detection test. Rather than using videos of real world driving scenes in which there is little control of when and where hazards appear, we based our test on a pedestrian detection task we previously developed for our driving simulator, which was shown to be sensitive to detection deficits in hemianopia, partial hemianopia, and central visual field loss (Bowers et al., 2009; Bronstad et al., 2013; Bronstad et al., 2011).

In this preliminary study, we evaluated whether a prototype version of the new video test would provide a robust measure of detection performance for people with hemianopia and quadranopia (loss of the same quarter of the field of vision in both eyes). We predicted that detection performance would be worse (lower detection rates and longer reaction times) for pedestrians appearing on the blind side than the seeing side, and that blind-side detection performance of hemianopes would be worse than that of quadranopes. In addition, we evaluated test-retest repeatability for the video test and examined preliminary validity by comparing detection performance on the video test to detection performance on the same task in the driving simulator. We expected that the video test performance would be predictive of driving simulator performance, but that detection rates would be lower and reaction times longer for the more complex, more interactive and cognitively-demanding driving simulator task.

\section{METHODS}

\section{Participants and Procedures}

Participants. Participants were recruited from the pool of visually-impaired volunteers who had previously participated in studies at Schepens. Inclusion criteria were: hemianopia or quadranopia on conventional visual field testing (Goldmann perimeter); binocular visual acuity of 20/40 or better with habitual correction; no significant cognitive decline (Mini Mental State Examination $\geq 24$ ); and no visual neglect (Bells Test and Schenkenberg Line Bisection Test). Data are reported for 13 participants (7 hemianopes, 6 quadranopes) with a median age of 59 years (range 19 to 83). Stroke was the main cause of the field loss (9 patients). Participants each completed one driving simulator session and two video detection test sessions. The study was approved by the institutional review board at Schepens.

Pedestrian detection task. The same pedestrian detection task was used for the video test and the driving simulator test. In brief, life-size pedestrian figures $(1.8 \mathrm{~m}$ tall, wearing a white shirt and blue trousers; Figure 1) appeared at random intervals and walked or ran (exhibiting salient biological motion) toward the roadway, as if to cross the road (Bronstad et al., 2013). The pedestrians were programmed such that there would have been a collision if the virtual vehicle had continued at the posted speed limit and the virtual pedestrian had entered the travel lane. However, pedestrians stopped at the edge of the travel lane. A total of 60 pedestrians were presented (30 on the right and 30 on the left), appearing with equal frequency at small $\left(4^{\circ}\right)$ and 
large $\left(14^{\circ}\right)$ eccentricities. Participants pressed a response button (video test) or the horn (driving simulator) as soon as they detected a pedestrian. They were allowed to make natural head and eye movements throughout the task, but were not given any instructions about specific head or eye movement scanning strategies.

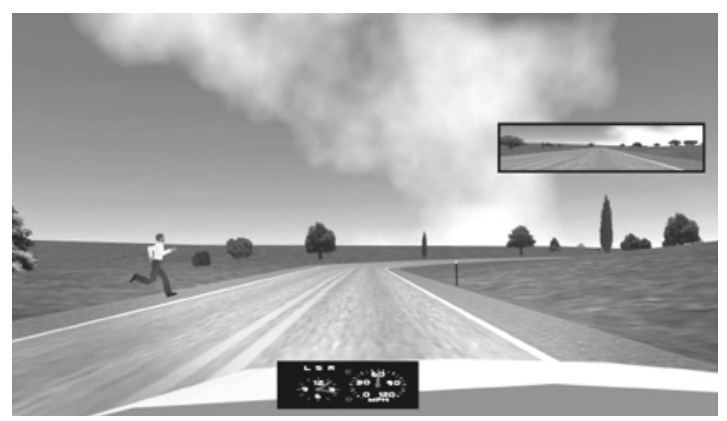

Figure 1. Screen shot of pedestrian detection task

Driving simulator test. Participants completed five drives ( 3 in the city and 2 on the highway), each about 10 minutes, in a FAAC PP-1000 driving simulator (with $225^{\circ}$ horizontal field of view, a motion seat, and controls typical for an automatic transmission car). They were instructed to follow the rules of the road and control the vehicle speed and steering (in addition to pressing the horn when a pedestrian was seen). Eye and head movements were recorded with a 6-camera remote infra-red Smart-Eye Pro 5 tracking system (Goteborg, Sweden).

Creating the video test. Videos were recorded directly from the central screen of the driving simulator while an investigator, familiar with the simulator and scenarios, drove a series of city and highway drives at the posted speed limit and in the center of the lane to ensure that there were no cues as to when pedestrians appeared and that they appeared at the expected eccentricities. Each recording was about 10 minutes in duration with 12 pedestrian appearances. While 10 minutes was appropriate for each simulator test drive, it was too long for the video detection test. Segments were therefore extracted from the recordings to create five (3 city and 2 highway) shorter, 3-minute videos using VideoPad Video Editor (NCH Software, Canberra, Australia, v 2.41). Stops and turns were excluded to reduce the potential for motion sickness. Each short video included 12 segments with pedestrians and 5 to 9 segments without pedestrians. The time between pedestrian appearances ranged from 7 to 30 seconds. Within each video, there were equal numbers of pedestrians on each side (right/left) and at each eccentricity (small/large).

Administering the video test. Participants sat $1.3 \mathrm{~m}$ away from a large rear-projection screen. A custom program played each 3-minute video continuously and recorded the time at which the button was pressed. At this distance the screen subtended the same visual angle as the central screen of the driving simulator ( $65^{\circ}$ horizontal by $40^{\circ}$ vertical). Eye and head movements were not recorded.

\section{Data Analysis}

Responses recorded during the experimental sessions were used to determine detection rates and reaction times (difference between pedestrian appearance time and participant response time). Nonparametric statistics (Wilcoxon Signed Ranks and MannWhitney U tests) were used to 
examine the effects of visual field loss and pedestrian eccentricity on detection rates and reaction times. Test-retest repeatability was quantified in terms of the difference in detection performance between the two video test sessions. The coefficient of repeatability is usually given as twice the standard deviation of the mean of the differences, i.e. the $95 \%$ confidence limit (Bland \& Altman, 1986). However, as the difference data were not normally distributed, we expressed the coefficient of repeatability as half of the range between the 5th and 95th percentiles. Spearman's rho was used to quantify the relationship between video test performance and driving simulator performance.

\section{RESULTS}

\section{Video Test: Effect of Visual Field Loss}

Detection rates were significantly lower on the blind than the seeing side (medians $77 \%$ and $100 \%$ respectively; $\mathrm{z}=2.5, \mathrm{p}=0.012$; Figure 2 ), and reaction times were significantly longer (medians 0.93s and 0.79s; $\mathrm{z}=2.8, \mathrm{p}=0.005$; Figure 2). This analysis was for data from all participants pooled across small and large eccentricities. In addition, we examined the effects of eccentricity (small or large) and amount of field loss (hemianopia or quadranopia) on blind-side detection performance. There was a trend for hemianopes to have more impaired blind-side detection performance than the quadranopes (lower detection rates and longer reaction times; Figure 2); however, there was a wide range of performance in both groups, especially at large eccentricities. Both hemianopes and quadranopes had lower detection rates and longer reaction times at the large than the small eccentricity on the blind side (Figure 3); however, the detection rate differences were noticeably greater for the hemianopes than the quadranopes (Figure 3).
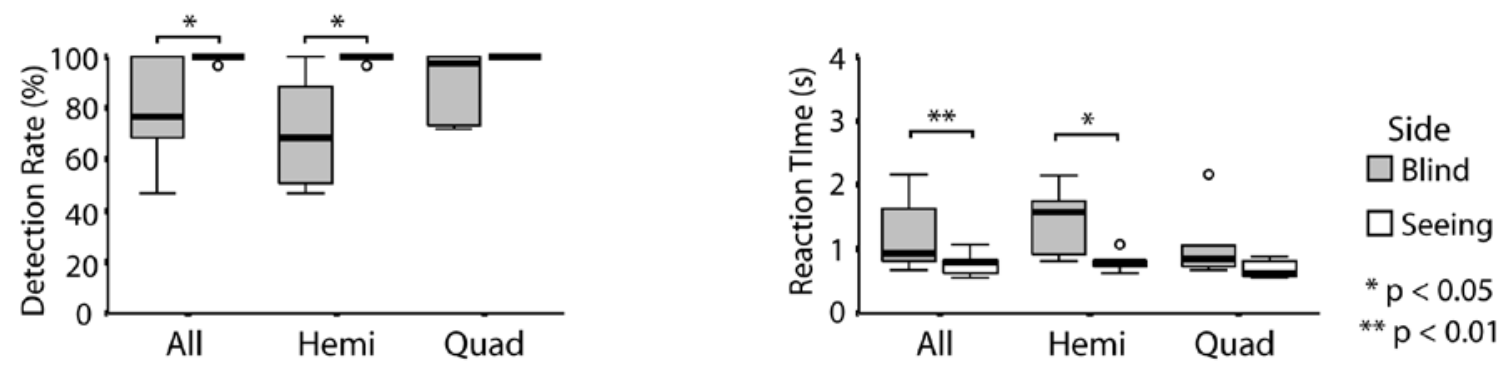

Figure 2. Median detection rates and reaction times on blind and seeing sides Detection rates were lower and reaction times longer on the blind than the seeing side.

Data are collapsed across small and large eccentricities. Open circles are outliers
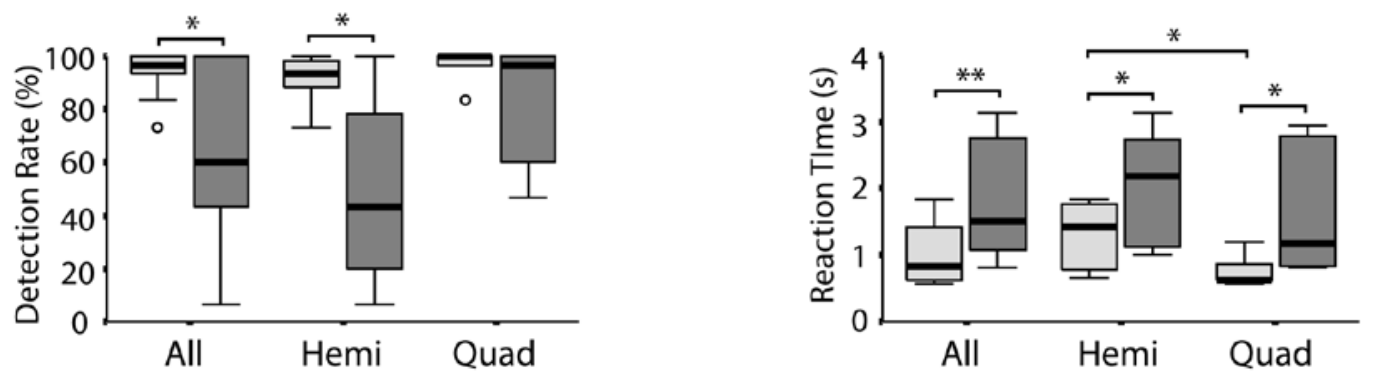

Eccentricity

$\square$ Small

$\square$ Large

${ }^{*} \mathrm{p}<0.05$

${ }^{* *} p<0.01$

Figure 3. Blind-side detection rates and reaction times for small and large eccentricities Detection rates were lower and reaction times longer at the large eccentricity 


\section{Video Test-Retest Repeatability}

Overall, there was no significant between-session differences in detection rates for either the blind side (median difference $0 \%, \mathrm{z}=1.05, \mathrm{p}=0.293$; Figure 4 , left) or the seeing side (constant at $100 \%$ for both sessions). The coefficient of repeatability was $10 \%$ for blind side detection rates; only 4 participants had an absolute between-session difference of more than $5 \%$. There was a trend for blind-side reaction times to be slightly faster at the second session (median difference 0.08s, $\mathrm{z}=1.77, \mathrm{p}=0.077$; Figure 4, middle), but there was no between-session difference for seeing-side reaction times (median 0.03s, $\mathrm{z}=0.83, \mathrm{p}=0.409$; Figure 4 , right). The coefficient of repeatability was $0.62 \mathrm{~s}$ for blind-side reaction times and 0.28 s for seeing-side reaction times. For eight participants, blind-side reaction times at the second session were within $0.4 \mathrm{~s}$ of those at the first session; however there were four notable outliers with between-session differences $\geq 0.5 \mathrm{~s}$ (Figure 4, middle).
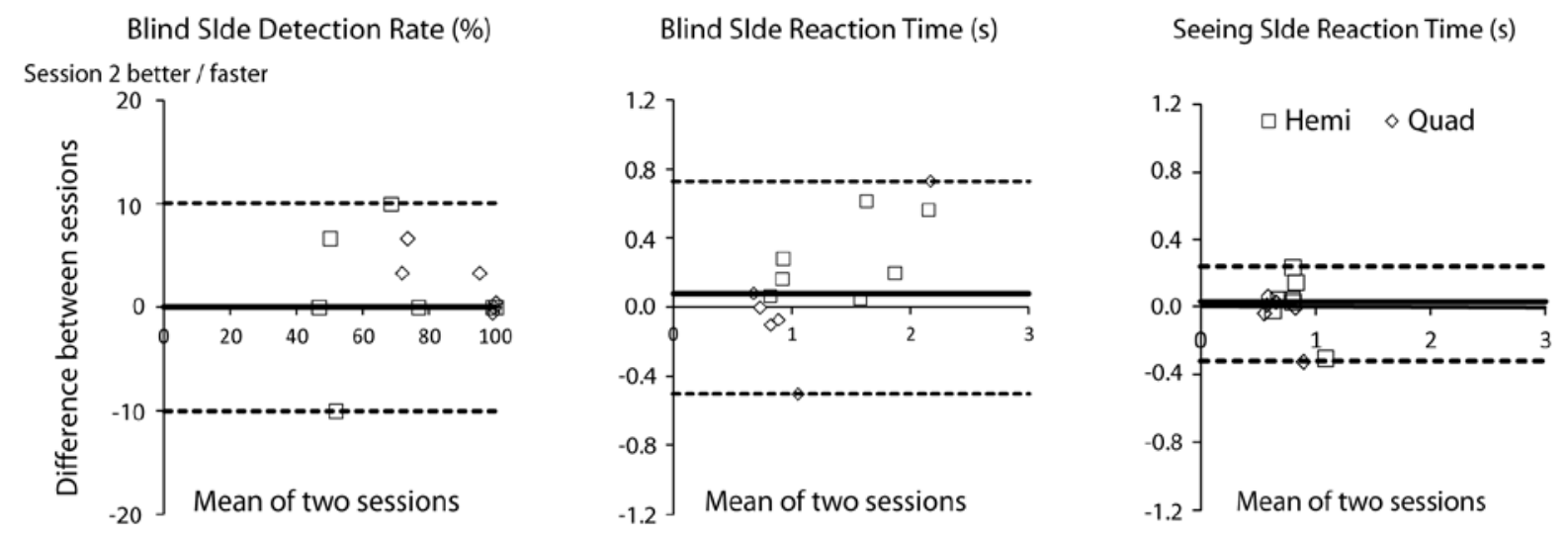

Session 1 better / faster

Figure 4. Differences in detection performance between the two video test sessions The solid line is the median of the differences. The dashed lines are the $5^{\text {th }}$ and $95^{\text {th }}$ percentiles

\section{Comparison to Driving Simulator}

For the comparison of video test and driving simulator performance, detection rates and reaction times were averaged across the two video test sessions. Higher blind-side detection rates on the video test were associated with higher blind-side detection rates in the driving simulator (rho = $0.78, p=0.002$; Figure 5, left). However, there was wide between-subject variability, with some participants having better detection rates in the video test and others having better detection rates in the driving simulator. Overall, there was no significant difference in detection rates between the video and simulator test (medians, $77 \%$ and $85 \%$, respectively, $\mathrm{z}=0.3, \mathrm{p}=0.767$ ). Similarly, longer reaction times in the video test were associated with longer reaction times in the driving simulator (rho $=0.60, p=0.03$; Figure 5 , right). However, as expected, reaction times were shorter on the video test than in the driving simulator $(0.93 \mathrm{~s}$ and $2.67 \mathrm{~s}$, respectively, $\mathrm{z}=3.2 \mathrm{p}=$ 0.001). Again, there was wide between-subject variability. 

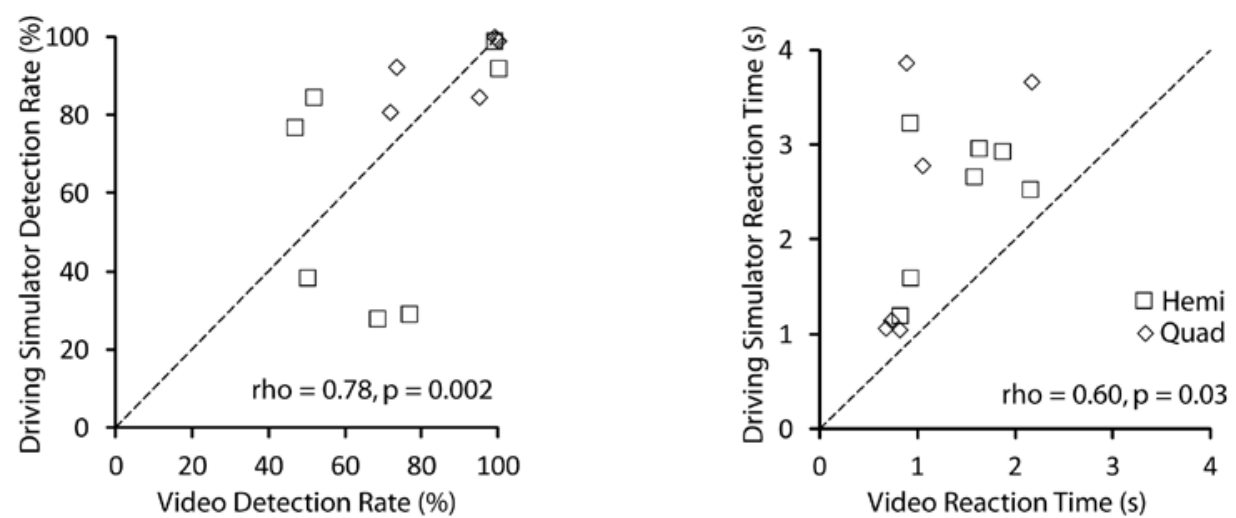

Figure 5. Blind-side detection performance for the video and driving simulator tests for each participant The diagonal dashed line $(\mathrm{y}=\mathrm{x})$ is for reference purposes only

\section{DISCUSSION}

Our video detection test was sensitive to detection deficits in both hemianopia and quadranopia. Detection rates were lower and reaction times longer on the blind than seeing sides with the deficits being greater at large than small eccentricities. In agreement with prior studies using more complex simulated driving tasks (Bowers et al., 2009; Iorizzo et al., 2011; Papageorgiou et al., 2012), there was a wide range of performance within both groups. This suggests that, even though the video test involved only watching videos without the higher cognitive load or interactivity of a driving simulator test, it was still sensitive enough to capture between-subject differences in the ability to compensate for hemianopic and quadranopic visual field loss, a variability that is not captured by conventional perimetry.

In general, detection rate repeatability was good. However, four participants did demonstrate relatively large ( $>0.5 \mathrm{~s}$ ) between-session differences in blind-side reaction times (Figure 4, middle). One had slower reaction times at the second session suggesting fatigue effects, while three had faster reactions, suggesting that they might have been using a more efficient scanning strategy at the second session. As this was a pilot study, eye movements were not recorded during the video test sessions and we could not formally test this hypothesis.

Participants with better blind-side detection rates and faster reaction times on the video test tended to have better blind-side detection rates and faster reaction times in the driving simulator, providing preliminary evidence in support of the validity of the video test. As expected, reaction times were significantly faster for the video than the driving simulator test as participants did not have to steer the vehicle or perform any other driving tasks. Although median detection rates were not significantly different for the two tests, there were two participants with low driving simulator detection rates (about 30\%) who had much higher detection rates $(70-80 \%)$ in the video test (Figure 5, left). These participants demonstrated little blind-side scanning in the driving simulator. It is likely that they scanned more frequently to the blind side during the video test, as it was much less demanding than driving in the simulator.

In summary, these preliminary data suggest our video test provides a simple method of measuring detection ability relevant to driving in people with hemianopia and quadranopia. It takes only 15 minutes to administer and, in the future, could be implemented on a desktop computer and large TV monitor. Furthermore, it did not cause any participant discomfort (unlike 
driving simulator tests which may be uncomfortable for up to $25 \%$ of participants). We are continuing to develop and refine the test, including adding a secondary task that will simulate the additional cognitive demands and divided attention conditions of real world driving.

\section{ACKNOWLEDGEMENTS}

Funded in part by TATRC Military Vision Research Program, Proposal 11066002 (subtask 10), and NIH grants EY018680, T35EY007149, EY12890, and P30EY003790. The authors thank Alex Hwang, Henry Apfelbaum, Jih-Ping Chern, and Robert Goldstein for technical assistance with programming, video editing, and development of analysis software.

\section{REFERENCES}

Bland, J. M., \& Altman, D. G. (1986). Statistical methods for assessing agreement between two methods of clinical measurement. Lancet, 1(8476), 307-310.

Bowers, A. R., Mandel, A. J., Goldstein, B., \& Peli, E. (2009). Driving with hemianopia: 1. Detection performance in a simulator. Investigative Ophthalmology and Visual Science, 50, 5137-5147.

Bronstad, P. M., Bowers, A. R., Albu, A., Goldstein, R. B., \& Peli, E. (2013). Driving with central visual field loss I: Impact of central scotoma on response to hazards. JAMA Ophthalmology, Published online January 17, 2013. doi:10.1001/jamaophthalmol.2013.1443.

Bronstad, P. M., Bowers, A. R., Albu, A., Goldstein, R. G., \& Peli, E. (2011). Hazard detection by drivers with paracentral homonymous field loss: A small case series. Journal of Clinical \& Experimental Ophthalmology, S5:001.doi: 010.4172/2155-9570.S4175-4001.

Dow, J. (2011). Visual field defects may not affect safe driving. Traffic Injury Prevention, 12(5), 483-490.

DVLA Drivers Medical Group. (2011). For medical practitioners. At a glance guide to the current medical standards of fitness to drive. Swansea, UK: Driver Vehicle Licensing Authority.

Iorizzo, D. B., Riley, M. E., Hayhoe, M., \& Huxlin, K. R. (2011). Differential impact of partial cortical blindness on gaze strategies when sitting and walking - An immersive virtual reality study. Vision Research, 51(10), 1173-1184.

Papageorgiou, E., Hardiess, G., Ackermann, H., Wiethoelter, H., Dietz, K., Mallot, H. A., \& Schiefer, U. (2012). Collision avoidance in persons with homonymous visual field defects under virtual reality conditions. Vision Research, 52(1), 20-30.

Peli, E. (2002). Low Vision Driving in the USA: who, where, when and why. CE Optometry, 5(2), 54-58.

Yazdan-Ashoori, P., \& ten Hove, M. (2010). Vision and Driving: Canada. Journal of NeuroOphthalmology, 30(2), 177-185. 